\title{
Successful adaptation of fever and neutropenia clinical practice guideline in China
}

\author{
Alicia Koo ${ }^{1,2}$, Changgang Li ${ }^{4}$, Liwei Linda Liư ${ }^{4}$, Huirong Mai ${ }^{4}$, Feiqiu Wen ${ }^{4}$, Paula D. Robinson ${ }^{5}$, L. Lee Dupuis ${ }^{2,3}$ \\ and Lillian Sung ${ }^{1,3^{*}}$ (1)
}

\begin{abstract}
Background: To describe a process for adapting a supportive care clinical practice guideline (CPG) for use in a middle income country setting.

Method: We reviewed different approaches for CPG adaptation and created a straight-forward approach for adapting supportive care guidelines for use in Shenzhen, China. The initial CPG to be adapted was for the empiric management of fever and neutropenia (FN) in children with cancer and hematopoietic stem cell transplantation recipients.

Results: The steps to be used in adaptation were as follows: review of local guideline; understanding local clinical pathways and contexts through interviews; development of worksheets to facilitate adaptation decisions; deliberation of guideline recommendations in focus groups; and drafting of the adapted FN CPG. After several iterations, stakeholders agreed upon a final adapted guideline.
\end{abstract}

Conclusions: We described an approach to adaptation of a supportive care CPG for the middle income country setting of Shenzhen, China. Although we believe this work has broad applicability, this approach requires rigorous evaluation, both in terms of methodology and the validity of the adapted guideline. Future work will evaluate implementation of the adapted CPG.

\section{Background}

Clinical practice guidelines (CPGs) are important to facilitate provision of high quality evidence-based clinical care. Studies have demonstrated that compliance with guidelines can improve patient outcomes [1-3]. For children with cancer, CPGs to address supportive care issues are particularly important since cancer treatment approaches in pediatric cancer are primarily driven by clinical trials whereas supportive care is highly variable $[4,5]$.

CPGs are defined as "statements that include recommendations intended to optimize patient care that are informed by a systematic review of evidence and an assessment of the benefits and harms of alternative care options" [6]. Much attention has been directed at

\footnotetext{
*Correspondence: Lillian.sung@sickkids.ca

'Division of Haematology/Oncology, The Hospital for Sick Children, 555

University Avenue, Toronto, ON M5G 1X8, Canada

${ }^{3}$ Program in Child Health Evaluative Sciences, The Hospital for Sick Children,

555 University Ave, Toronto, ON M5G 1X8, Canada

Full list of author information is available at the end of the article
}

specifying the methodology required to create robust or trustworthy guidelines and these principles have been articulated by the Institutes of Medicine [6, 7]. Several pediatric cancer groups are focused on the development of trustworthy pediatric cancer supportive care guidelines including the Pediatric Oncology Group of Ontario, the C17 Council, The American Society of Pediatric Hematology and Oncology, the Dutch Children's Oncology Group and the Children's Cancer Leukemia Group. These groups have been brought together under the umbrella organization International Pediatric Oncology Guidelines in Supportive Care Network (iPOG Network; http://www.sickkids.ca/Research/iPOG/). These groups are all primarily focused on creating CPGs applicable to the high income country (HIC) setting.

While creation of CPGs for HICs is an important goal, most children with cancer live in low and middle income countries (LMICs) [8]. There may be a desire to adapt CPGs developed for HICs for LMICs since those institutions may not have the resources for de novo CPG 
development. Guideline adaptation is defined as a systematic approach to the modification of a source guideline for application in a different context [9]. Guideline adaptation requires fewer resources in comparison to de novo guideline development. In this case, considerations for adaptation must include applicability of the knowledge base, similarity in values and preferences, availability of resources such as tests and medications, and costs.

Adaptation of CPGs through a formal process is important to ensure that the adaptation process does not undermine the validity of the recommendations made by the source CPG and to promote uptake by making stakeholders a part of the adaption process [10]. In spite of the great need to adapt supportive care CPGs to LMICs, we found a dearth of information on this topic to direct guideline developers and clinicians on how to accomplish this goal. Thus, the purpose of this article is to describe our thought process and experience with adaptation of a pediatric cancer CPG focused on fever and neutropenia (FN) for the middle income country setting of Shenzhen, China.

\section{Methods}

Through an initiative with The Hospital for Sick Children's (SickKid's) International office and a partnership with the Shenzhen Children's Hospital, we began a program to adapt supportive care guidelines. We began with FN since this common side effect of therapy is responsible for considerable morbidity, mortality, costs and resource utilization. The source CPG was that by Lehrnbecher and colleagues [11] and it was chosen because it is specific to pediatric cancer and it has been endorsed by multiple organization including the Children's Oncology Group, American Society of Pediatric Hematology and Oncology, Pediatric Oncology Group of Ontario, C17 Council, American Society of Clinical Oncology and Multinational Association of Supportive Care in Cancer.

The source CPG used the Grading of Recommendations, Assessment, Development and Evaluations (GRADE) approach to make recommendations [7, 12]. With this approach, a strong recommendation is made when benefits clearly outweigh risks or vice versa. When a strong recommendation is made, almost all patients should receive the recommended intervention as a matter of policy. In contrast, when a weak recommendation is made, the benefits and risks of the intervention may be closely matched or there may be considerable uncertainty about the magnitude of the benefits and risks [7, 12]. When weak recommendations are made, facilities can elect to institute or not institute the intervention depending on local factors, preferences and values, or facilities may enable children and families to make decisions based upon their own perspectives. In addition to making strong or weak recommendations, the GRADE approach classifies evidence as high, moderate, low or very low based upon the degree of certainty in the estimates of benefits and risks for the target population [13].

\section{Setting}

The setting of CPG adaptation and implementation was Shenzhen Children's Hospital. This hospital was established in 1998 and is located in Shenzhen, China, immediately north of Hong Kong. It is a public hospital affiliated with Shantou University Medical College. There were 167 new pediatric cancer cases diagnosed in this center in 2015.

\section{Identification of guideline adaptation approaches}

The first step was to identify approaches to guideline adaptation or implementation which might be helpful in our setting. We did not conduct a systematic review of adaption approaches but rather, focused on the ones we had used in our other guideline-related work.

\section{ADAPTE [10]}

This approach has been widely used to adapt guidelines to other settings. This approach consists of three main phases, namely set-up, adaptation and finalization. During the set-up phase, the tasks are outlined. During adaptation, the adapted guideline is prepared after assessing evidence and guideline quality, currency, content and applicability and making decisions around adaptation. During the final phase, feedback on the adapted CPG is obtained. We modified the original step-by-step process because of the limited time and resources available to perform guideline adaptation in this context.

\section{Queen's University Research Roadmap for Knowledge Implementation (QuRKI) [14]}

This approach is focused on ensuring that the best available evidence is integrated into practice and has three major phases, namely issue identification/clarification, solution building and implementation, evaluation and nurturing the change.

\section{Checklist of determinants of practice [15]}

The authors used a systematic review and a consensus process to develop a checklist of determinants of healthcare practice. They propose that use of such a checklist can improve implementation of a specific change in practice.

\section{Results}

After selecting the source guideline, we adapted the source pediatric FN CPG to the Shenzhen context using the following steps (Fig. 1). 


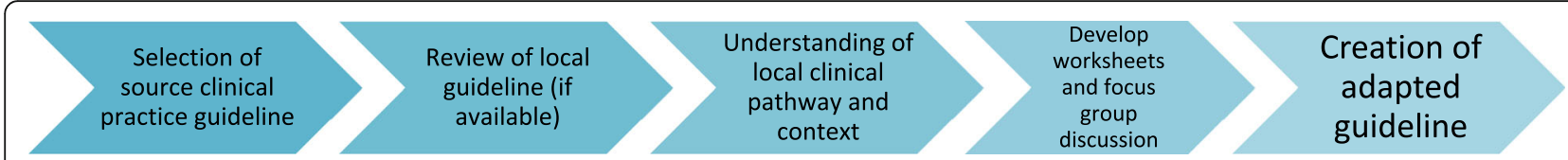

Fig. 1 Steps Taken to Clinical Practice Guideline Adaptation

1. Review of Local Guideline(s) (if available): In anticipation of the visit to Shenzhen Children's Hospital, we confirmed that a current, written local FN guideline existed and reviewed its recommendations. We identified the recommendations which were consistent or inconsistent with the source CPG and color coded the recommendations as follows: green (consistent with source CPG), yellow (inconsistent with source CPG weak recommendations) and red (inconsistent with source CPG strong recommendations).

2. Understanding of Local Clinical Pathway and Context: In attempting to better understand the local context, we arranged a series of interviews with local healthcare providers and stakeholders. First, we met with pediatric oncology clinicians to better understand the local healthcare system and to understand the clinical pathway for different types of patients with FN including inpatients and outpatients. Next, we conducted interviews with the following services: microbiology, infection control, the intensive care unit, emergency department, pharmacy and information technology. While many other services could also impact on the FN CPG, we were constrained by team availability and time limitations.

3. Development of Worksheets to Facilitate Adaptation Decisions: Using the reviewed approaches (ADAPTE, QuRKI and the Checklist of Determinants of Practice), we developed a worksheet to guide the adaptation discussion (Table 1 shows the final modified version using an example recommendation). A separate worksheet was generated for each recommendation in the source CPG. The document headings included the source CPG recommendation; the local recommendation; concordance with source CPG; and supports required to implement the source CPG recommendation. A decision regarding the appropriateness of the source CPG recommendation to the local setting was listed next; this decision was made during the focus group which is described in the next step. If this decision was "inappropriate", then discussion would stop for that recommendation. If the decision was "appropriate", then discussion would continue with the goal of understanding local factors important to successful implementation.

4. Focus Group to Deliberate Adapted Guideline Recommendations: After gaining some insight into

the local context, we conducted a focus group with the following participants: all pediatric oncology clinicians, relevant administrators, pharmacy, nursing leadership, and infection control personnel. The session was scheduled for $4 \mathrm{~h}$ and began with education focused on CPGs to explain what constitutes a robust CPG according to the Institutes of Medicine [6] and to delineate the GRADE approach to recommendation development $[7,12]$.

Next, we reviewed each section of the source CPG sequentially: initial presentation of $\mathrm{FN}$, ongoing management and empiric antifungal therapy. The original questions from the source guideline were used without modification. We first presented a color-coded comparison of local guideline and source CPG recommendations. We then reviewed each source CPG recommendation using the worksheet shown in Table 1. The following headings were completed prior to the focus group: the source CPG recommendation, the local recommendation, concordance with source $\mathrm{CPG}$, and supports required to implement the source CPG recommendation.

The remaining elements of the worksheet were completed during the focus group and decisions were projected in real time to ensure that focus group participants understood and agreed with decisions being made during the session. In the original worksheet, these elements were the appropriateness of the source CPG

Table 1 Example Worksheet To Facilitate Adaptation Decisions ${ }^{a}$

Source Guideline

Recommendation

Local Recommendation

Concordance

Required Supports

Appropriate to Setting?

If no, then stop

Facilitators and Barriers

Resources Required

including Patient Costs

Other Comments

aTable is populated up to "Required Supports" prior to focus group and remaining fields are completed during the focus group. A table is generated for each recommendation from the source clinical practice guideline
Do not modify the initial empiric antibacterial regimen based solely on persistent fever in children who are clinically stable (Strong recommendation, Low quality evidence)

After using cefepime for 7 days, change the dose to $1500 \mathrm{mg} / \mathrm{m} 2 /$ dose (MAX $2000 \mathrm{mg}$ ), IV every $12 \mathrm{~h}$

No

Ability to judge clinical stability 
recommendation to the local setting; facilitators and barriers; incentives to implementation; resources required including institutional and patient costs; organizational priority for change; and effort and feasibility.

We reviewed practices which were consistent with the source CPG briefly in order to ascertain the perceived degree of compliance with the local CPG recommendation and whether there were barriers to compliance. Next, we reviewed the inconsistent recommendations and identified whether each recommendation was appropriate to the setting. Strong recommendations of the source CPG which were discordant with local clinical pathways (color-coded red) were prioritized for discussion.

Working through each recommendation sequentially and using the worksheet (Table 1) to guide the discussion, we achieved consensus on each recommendation in the adapted guideline. For consensus recommendations which differed from the current local clinical pathway, we determined the resources required to achieve change.

5. Drafting of Adapted Pediatric FN Guideline: Based upon the focus group discussion, we drafted the adapted FN CPG and circulated it among the pediatric oncology clinicians and pharmacists in Shenzhen. After several iterations, we agreed upon a final document. The edits primarily focused on clarifications rather than deviating from decisions made during the focus group.

These steps were completed successfully. All but one of the strong recommendations from the source CPG were included in the adapted guideline. Where the source CPG recommends either caspofungin or liposomal amphotericin $\mathrm{B}$ for empiric antifungal therapy, the adapted CPG recommends oral voriconazole for empiric anti-fungal therapy since in Shenzhen, caspofungin is expensive and liposomal amphotericin B is not available. All other strong recommendations of the source CPG were incorporated into the adapted guideline.

The major changes to the local guideline and to clinical practice were as follows: (1) Standardization of the front-line antibiotic regimen for patients with and without sepsis; (2) Reservation of vancomycin and a carbapenem for clinical (hemodynamic instability or clinical evidence of a specific infection type) or microbiological indications and reassessment and deescalation at $48-72 \mathrm{~h}$ if appropriate; (3) Discontinuation of broad-spectrum antibiotics with evidence of neutrophil recovery in the absence of positive cultures, fever, or ongoing clinical site of infection; (4) Not changing antibiotic coverage for persistent fever alone; and (5) Discontinuation of routine beta-Dglucan testing.
We found that while feasible, the worksheet included some questions which were less useful and thus, we removed organizational priority; efforts and feasibility; and implementation incentives in the final worksheet (Table 1).

\section{Discussion}

We have described a process to adapt a supportive care CPG for a middle income country setting. This process is important; most children with cancer live in LMICs and supportive care is at least as important in these centers as in HICs [16]. We present our experience in order to help others who plan to adapt CPGs for LMIC utilization.

In this experience, we capitalized on both an existing CPG and the availability of a local guideline. In the absence of a local guideline, the worksheet could still be used although local recommendations would be replaced by the most common local practice. More problematic is how to adapt guidelines in the absence of robust CPGs for a specific topic, a common occurrence in pediatric cancer [17]. In this case, we would suggest first identifying if robust CPGs exist on the topic in a similar population such as adults with cancer. In the absence of such a CPG, the options include de novo guideline development if recent systematic reviews are available or adaptation using consensus recommendations if they exist.

Our future plans include evaluating implementation of the adapted guideline and beginning to evaluate outcomes before and after implementation. We recommend that implementation be sensitive to local practices and culture and that the process be facilitated by frequent contact with the adaptation team. We also plan to adapt other supportive care CPGs. A major limitation to CPG adaptation is the absence of robust CPGs in many areas of pediatric cancer supportive care [17]. However, we anticipate that the recent formation of the iPOG Network will begin to bridge this gap.

The strengths of the proposed approach are the careful planning of the adaptation process; the utilization of existing frameworks; and its successful application in a real-life setting. Weaknesses include its resourceintensive nature and due to challenges with language, the requirement to translate all documents between English and Chinese. Moderators of the guideline process also need to be very familiar with the source CPG to be able to adjudicate the appropriateness of recommendation deviation. Another important limitation is that we did not strictly follow every step of the original ADAPTE process because of constraints with time and resources. This deviation could affect the robustness of the adapted product. 


\section{Conclusion}

We described an approach to adaptation of a supportive care CPG for the middle income country setting of Shenzhen, China. Although we believe this work has broad applicability, this approach requires rigorous evaluation, both in terms of methodology and the validity of the adapted guideline. Future work will evaluate implementation of the adapted CPG.

\section{Abbreviations}

CPG: Clinical Practice Guideline; FN: Fever and Neutropenia; GRADE: Grading of Recommendations, Assessment, Development and Evaluations; HIC: High Income Country; iPOG Network: International Pediatric Oncology Guidelines in Supportive Care Network; LMIC: Low and Middle Income Countries; QuRKI: Queen's University Research Roadmap for Knowledge Implementation

\section{Acknowledgements}

None.

\section{Funding}

There was no funding source for this study.

\section{Availability of data and materials}

Not applicable.

\section{Authors' contributions}

All authors have participated in the concept and design, data analysis and interpretation, drafting or revising of the manuscript. All authors read and approved the final manuscript.

\section{Ethics approval and consent to participate}

This study was approved by the Research Ethics Boards at both contributing institutions, the Hospital for Sick Children in Canada, and Shenzhen Children's Hospital in China.

\section{Consent for publication}

Not applicable.

\section{Competing interests}

The authors declare that they have no competing interests.

\section{Publisher's Note}

Springer Nature remains neutral with regard to jurisdictional claims in published maps and institutional affiliations.

\section{Author details}

'Division of Haematology/Oncology, The Hospital for Sick Children, 555 University Avenue, Toronto, ON M5G 1X8, Canada. ${ }^{2}$ Department of Pharmacy, The Hospital for Sick Children, 555 University Ave, Toronto, ON M5G 1X8, Canada. ${ }^{3}$ Program in Child Health Evaluative Sciences, The Hospital for Sick Children, 555 University Ave, Toronto, ON M5G 1X8, Canada. ${ }^{4}$ Department of Haematology/Oncology, Shenzhen Children's Hospital, Yitian Rd, Shenzhen, Guangdong 7019, China. ${ }^{5}$ Pediatric Oncology Group of Ontario, 480 University Ave., Suite 1014, Toronto, ON M5G 1V2, Canada.

Received: 14 June 2017 Accepted: 26 September 2017

Published online: 07 December 2017

\section{References}

1. Wright JD, Neugut Al, Ananth CV, Lewin SN, Wilde ET, YS L, Herzog TJ, Hershman DL. Deviations from guideline-based therapy for febrile neutropenia in cancer patients and their effect on outcomes. JAMA Intern Med. 2013;173(7): 559-68.

2. Aapro M, Molassiotis A, Dicato M, Pelaez I, Rodriguez-Lescure A, Pastorelli D, Ma L, Burke T, Gu A, Gascon P, et al. The effect of guideline-consistent antiemetic therapy on chemotherapy-induced nausea and vomiting (CINV): the Pan European Emesis Registry (PEER). Ann Oncol. 2012;23(8):1986-92.
3. Wockel A, Kurzeder C, Geyer V, Novasphenny I, Wolters R, Wischnewsky M, Kreienberg $\mathrm{R}$, Varga $\mathrm{D}$. Effects of guideline adherence in primary breast cancer-a 5-year multi-center cohort study of 3976 patients. Breast. 2010;19(2):120-7.

4. Lehrnbecher T, Aplenc R, Rivas Pereira F, Lassaletta A, Caselli D, Kowalczyk J, Chisholm J, Sung L, Group SSCW. Variations in non-pharmacological antiinfective measures in childhood leukemia-results of an international survey. Haematologica. 2012;97(10):1548-52.

5. Lehrnbecher T, Ethier MC, Zaoutis T, Creutzig U, Gamis A, Reinhardt D, Aplenc $\mathrm{R}$, Sung $\mathrm{L}$. International variations in infection supportive care practices for paediatric patients with acute myeloid leukaemia. Br J Haematol. 2009:147(1):125-8.

6. Clinical Practice Guidelines We Can Trust. Washington, D.C.: Institute of Medicine, National Academies Press; 2011.

7. Guyatt GH, Oxman AD, Vist GE, Kunz R, Falck-Ytter Y, Alonso-Coello P, Schunemann HJ, Group GW. GRADE. An emerging consensus on rating quality of evidence and strength of recommendations. BMJ. 2008; 336(7650):924-6.

8. Gelband H, Sankaranarayanan R, Gauvreau CL, Horton S, Anderson BO, Bray F, Cleary J, Dare AJ, Denny L, Gospodarowicz MK, et al. Costs, affordability, and feasibility of an essential package of cancer control interventions in low-income and middle-income countries: key messages from Disease Control Priorities, 3rd edition. Lancet. 2016:387(10033):2133-44.

9. Harrison MB, Van Den Hoek J. CAN-IMPLEMENT@: A Guideline Adaptation and Implementation Planning Resource. In. Kingston Ontario: Queen's University School of Nursing and Canadian Partnership Against Cancer; 2012.

10. Harrison MB, Legare F, Graham ID, Fervers B. Adapting clinical practice guidelines to local context and assessing barriers to their use. CMAJ. 2010; 182(2):E78-84.

11. Lehrnbecher T, Phillips R, Alexander S, Alvaro F, Carlesse F, Fisher B, Hakim $H$, Santolaya M, Castagnola E, Davis BL, et al. Guideline for the management of fever and neutropenia in children with cancer and/or undergoing hematopoietic stem-cell transplantation. J Clin Oncol. 2012;30(35):4427-38.

12. Guyatt $G H$, Oxman $A D$, Kunz R, Falck-Ytter $Y$, Vist GE, Liberati A, Schunemann HJ, Group GW. Going from evidence to recommendations. BMJ. 2008;336(7652): 1049-51.

13. Brozek JL, Akl EA, Alonso-Coello P, Lang D, Jaeschke R, Williams JW, Phillips B, Lelgemann M, Lethaby A, Bousquet J, et al. Grading quality of evidence and strength of recommendations in clinical practice guidelines. Part 1 of 3. An overview of the GRADE approach and grading quality of evidence about interventions. Allergy. 2009;64(5):669-77.

14. Harrison MB, Graham ID. Roadmap for a participatory research-practice partnership to implement evidence. Worldviews Evid-Based Nurs. 2012; 9(4):210-20.

15. Flottorp SA, Oxman AD, Krause J, Musila NR, Wensing M, Godycki-Cwirko M, Baker R, Eccles MPA. Checklist for identifying determinants of practice: a systematic review and synthesis of frameworks and taxonomies of factors that prevent or enable improvements in healthcare professional practice. Implement Sci. 2013:8:35.

16. Rodriguez-Galindo C, Friedrich P, Alcasabas P, Antillon F, Banavali S, Castillo L, Israels T, Jeha S, Harif M, Sullivan MJ, et al. Toward the Cure of All Children With Cancer Through Collaborative Efforts: Pediatric Oncology As a Global Challenge. J Clin Oncol. 2015;33(27):3065-73.

17. Sung L. Priorities for quality care in pediatric oncology supportive care. J Oncol Pract. 2015;11(3):187-9.

Submit your next manuscript to BioMed Central and we will help you at every step:

- We accept pre-submission inquiries

- Our selector tool helps you to find the most relevant journal

- We provide round the clock customer support

- Convenient online submission

- Thorough peer review

- Inclusion in PubMed and all major indexing services

- Maximum visibility for your research

Submit your manuscript at www.biomedcentral.com/submit
Biomed Central 